\title{
Health services and mortality in developed countries: a comment
}

\author{
Klim McPherson
}

The article on pages $344-348$ is a wonderful example of confounding in epidemiological investigations of mortality, when the expectation would naturally have been that health care provision would be negatively associated with all cause mortality. This study calculates the correlation of mortality at different ages with the most obvious and measurable aggregated indices of health care and consumption inputs. Surprisingly, among the highest positive correlations found were those between doctors per thousand population and mortality at young ages. Even the association of cigarette consumption and mortality at ages 55-64 was not as strong as the correlation between numbers of doctors and perinatal mortality-usually thought to be a sensitive index of appropriate health care provision.

Department of Public Health and Policy, London School of Hygiene and Tropical Medicine,

Keppel Street,

London WC1E 7HT.

$\mathrm{K}$ McPherson

Correspondence to: Professor K McPherson.

Based on a comment first published in The

Epidemiological Imagination. Ashtwn I ed. Buckingham Oxford Cniversity Press, 1994.

\section{Uncertainties rather than answers}

While not answering many questions, this paper gives rise to uncertainties which may not have been acknowledged. Thus, epidemiology is as useful in illuminating complicated casual pathways in medicine as it is in raising questions whose importance might not otherwise have been appreciated.

\section{Impact of medical intervention}

These data were extracted from national data systems in around 1970. At that time mortality rates (from age 5 to 64 ) from causes deemed to be amenable to, that is preventable by, medical intervention had reduced by around threefold since 1950. However, mortality from causes thought not to be amenable to medical intervention had hardly changed at all. But much more importantly, these latter causes were then 20 times more common that the former. Of course they included common cancers and cardiovascular diseases, for which primary prevention might be more appropriate, but which still warrant much medical intervention.

Importance of other factors in mortality Hence a high correlation with doctors per thousand population might simply reflect a rational response to a perceived need resulting from a higher incidence of these causes, which would not affect mortality but might easily affect the quality of people's lives. Thus, the paper really brought home to roost the notion that medical care may not be a life saving panacea after all and that much needed to be done to enable interventions to have dramatic effects on mortality. Now, of course, the extent to which medical intervention can affect the mortality from common cancers and cardiovascular disease is beginning to be noticed in mortality rates and this is in no small measure attributable to Archie Cohcrane's legacy of insisting on understanding about effectiveness rather than making unwarranted assumptions. Large randomised trials allow small increments in effectiveness to be reliably understood, which benefit many.

\section{Primary care the greatest hope}

However, the ratio of mortality under 65 to these so called non-amenable deaths is now more than 20 times as common as for amenable deaths, because the latter have decreased faster still. Primary prevention remains the greatest hope but very difficult to achieve. 